\title{
Optical Suppression of Energy Barriers in Single Molecule-Metal Binding
}

\author{
Qianqi Lin ${ }^{1}$, Shu Hu${ }^{1}$, Tamás Földes ${ }^{2,3}$, Junyang Huang ${ }^{1}$, Demelza Wright ${ }^{1}$, Jack Griffiths ${ }^{1}$, Bart de \\ Nijs ${ }^{1}$, Edina Rosta ${ }^{2,3}$, Jeremy J. Baumberg1* \\ 1. Nanophotonics Centre, Department of Physics, Cavendish Laboratory, University of Cambridge, Cambridge, CB3 OHE, England, U.K. \\ 2. Department of Chemistry, King's College London, 7 Trinity Street, London, SE1 1DB, U.K. \\ 3. Department of Physics and Astronomy, University College London, London, WC1E 6BT, U.K.
}

Understanding the interactions between molecules and metal surfaces is of widespread importance in electrochemistry, sensing, medical imaging/targeting, molecular electronics and spintronics. Although many techniques have characterized the molecule-metal transient bonds, conflicting conclusions arise from their buried location and heterogeneity, while single-molecule probes are scarce. Confinement of optical fields to picometre length-scales around adatoms (termed picocavities [1], Fig.1a,b) has enabled tipenhanced and surface-enhanced Raman spectroscopies (TERS and SERS, Fig.1e) of single-molecules. However how adatoms change with molecule-metal interactions, with light (both key ingredients in photocatalysis), and how they link to picocavity formation in metal nanogaps is not understood.

In this work [2], we demonstrate how the molecule-metal opto-chemical interaction influences the formation and stabilisation of adatoms yielding picocavities, as well as adlayers resulting in flares [3]. Through statistics from different molecules across a range of laser powers and modelling by density functional theory (DFT), we show how the local polarisation of molecule-metal electrons is amplified by illumination, gradually eliminating the energy barrier for adatom extraction and subsequently binding molecule-to-metal, instead of photothermal heating at the surface (Fig.1c,d). We find rates $\propto$ $\exp \left\{-U_{f}(I) / k_{B} T\right\}$ where intensity $I$ suppresses the barrier, scaling as $U_{f}(I) \propto U_{f}^{0} / I$.

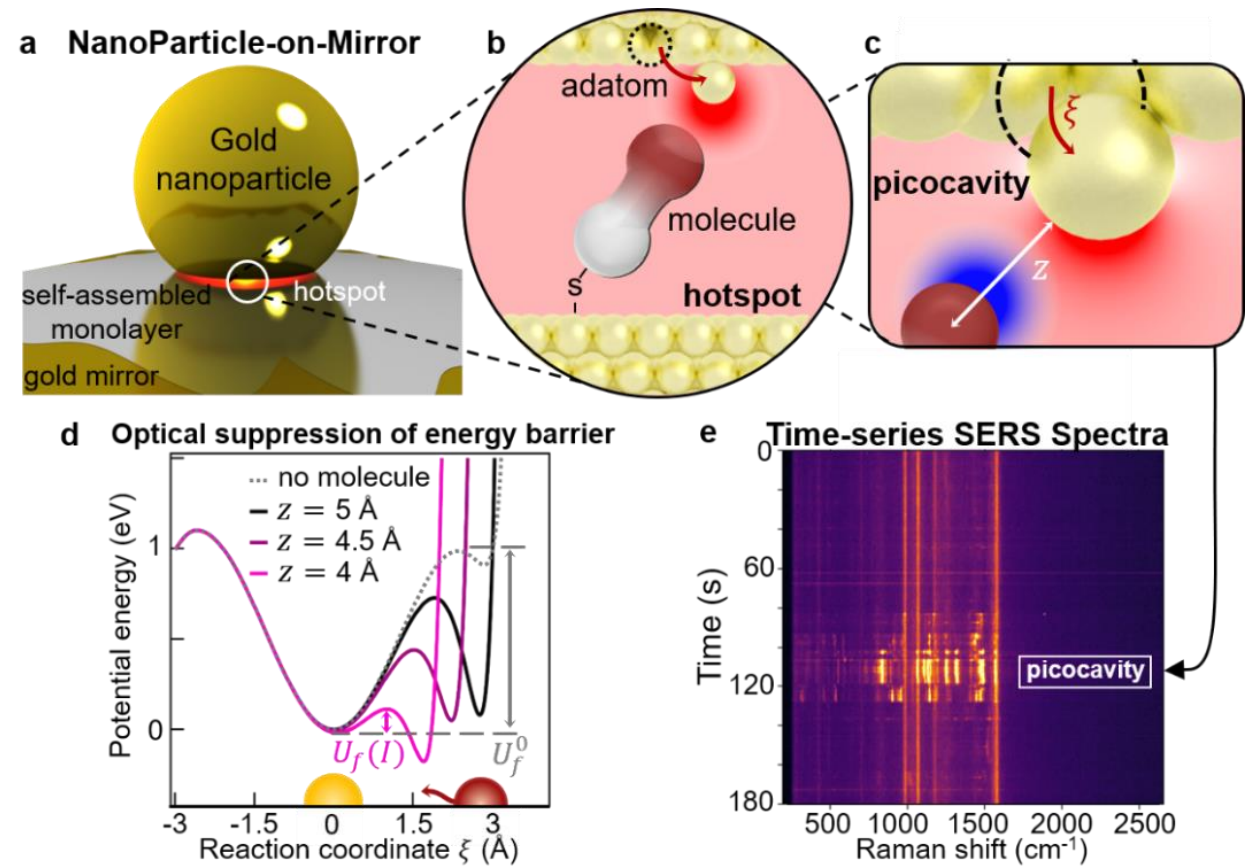

Fig. 1 a, Plasmonic nanocavity assembly, with optical field trapped in the hotspot. b,c, Scheme of a picocavity, with optical field (red) localised around adatom attracting the molecule tip. $\xi$ is reaction coordinate of adatom from initial site in facet, $z$ is molecule tip-adatom separation. d, Energy for picocavities when $z$ decreases by light (solid) vs without molecule (dashed). $U_{f}^{0}$ is barrier for adatom formation when laser is off, $U_{f}(I)$ is same barrier but at laser intensity $I$. e, Example of surface-enhanced Raman scattering (SERS) spectra for a picocavity.

Interactions between a polarizable atom and metallic atom create extremely powerful optical forces (>nN) capable of rearranging the material interface. This work provides not only a vital intuition for utilising lightmolecule-metal systems to control single-atom optical switches and semiconductor-metal optoelectronic devices, but also a strong spur to develop new theories capable of combining electromagnetism with quantum mechanics.

\section{References}

[1] F. Benz et al., "Single-molecule optomechanics in 'picocavities",, Science 354, 726 (2016).

[2] Q. Lin et al., “Optical Suppression of Energy Barriers in Single Molecule-Metal Binding”, under review (2021).

[3] C. Carnegie et al., "Flickering nanometre-scale disorder in a crystal lattice tracked by plasmonic flare light emission", Nat. Commun. 11, 682 (2020). 